\title{
Rectus Abdominis Endometrioma: A Rare Occurrence
}

\author{
Aravind Menon ${ }^{1}$, Alagesan Ganapathi ${ }^{2}$
}

\begin{abstract}
Endometriosis is defined as the presence of endometrial tissue outside the uterine cavity. Though the commonest site of occurrence is the pelvis, there have been cases of extrapelvic endometriosis reported in the literature. One of such rare sites is the rectus abdominis muscle, with only around 20 cases reported in the literature. Our patient was a 31-year-old female with a previous history of two cesarean sections who presented with cyclical pain over the scar. Imaging revealed the presence of endometrial tissue localized to the rectus abdominis muscle. Excision of the endometrioma with abdominal wall reconstruction using a polypropylene mesh was done. Herein, we present this case for its rarity with a short review of the literature on extrapelvic endometrioma.
\end{abstract}

Keywords: Endometriosis, Extrapelvic, Mesh, Rectus, Uterus.

Journal of South Asian Federation of Obstetrics and Gynaecology (2020): 10.5005/jp-journals-10006-1760

\section{INTRODUCTION}

Endometrioma is defined as the presence of hormonally responding endometrial tissue outside the uterine cavity and was first elaborated by Karl Freiherr von Rokitansky in 1860 as "adenomyoma." Common sites of occurrence are the pelvic organs, such as, ovaries, pouch of Douglas, and ligament supports of uterus, and rarely extrapelvic sites. Umbilicus, abdominal wall, vulva, appendix, thorax, and urinary tract are the common extrapelvic sites. The proposed theories of endometrioma are the metaplasia theory, induction theory, and transplantation theory that attributes the retrograde transport of endometrial tissue during menstruation. Patients usually present with cyclical pain during menstruation at the sites of implantation, especially the anterior abdominal wall. Abdominal wall endometriosis (AWE) can be diagnosed only with a strong clinical suspicion as it clinically mimics stitch granuloma, hematoma, and abscess. Our patient presented with cyclical pain at the site of a previous cesarean section, which on evaluation was found to be endometrioma confined to the rectus muscle, which is a rare site of occurrence of AWE with only around 20 cases reported in the literature. We present this case for its rarity in presentation and management.

\section{Case Description}

A 31-year-old female presented to the OPD with complaints of cyclical lower abdominal pain following menses. She had a previous history of two cesarean sections done, both children healthy at present, and also underwent laparoscopic sterilization after the birth of the second child. Three years after the last child birth, she started experiencing this symptom of abdominal pain localized to the cesarean scar site, for which she was prescribed pain killers only with partial symptom relief. On abdominal examination, she had a Pfannenstiel scar with localized scar tenderness and induration around the scar. On ultrasonogram screening, a well-defined soft tissue lesion of size $5.3 \times 2.5 \mathrm{~cm}$ was seen anterior to bladder and uterus, suggestive of endometrioma in the anterior abdominal wall. A contrast CT scan of the abdomen with MRI screening of the anterior abdominal wall showed a poorly defined soft tissue lesion with heterogeneous enhancement $(6 \times 3.3 \mathrm{~cm})$ present in the lower rectus abdominis muscle on both sides; the soft tissue
${ }^{1}$ Department of General Surgery, Stanley Medical College, Chennai, Tamil Nadu, India

${ }^{2}$ Department of General Surgery, Shanthi Nursing Home, Tirunelveli, Tamil Nadu, India

Corresponding Author: Aravind Menon, Department of General Surgery, Stanley Medical College, Chennai, Tamil Nadu, India, Phone: +918056180309, e-mail: aravindmenonk@gmail.com

How to cite this article: Menon A, Ganapathi A. Rectus Abdominis Endometrioma: A Rare Occurrence. J South Asian Feder Obst Gynae 2020;12(2):104-107.

Source of support: Nil

Conflict of interest: None

lesion had peripheral strands extending to the lower abdominal scar and to the anterior aspect of the uterus and urinary bladder (Figs 1 and 2). With the clinicoradiological diagnosis of the rectus muscle endometriosis, the patient was counseled for excision of the endometriotic tissue and total abdominal hysterectomy. Under

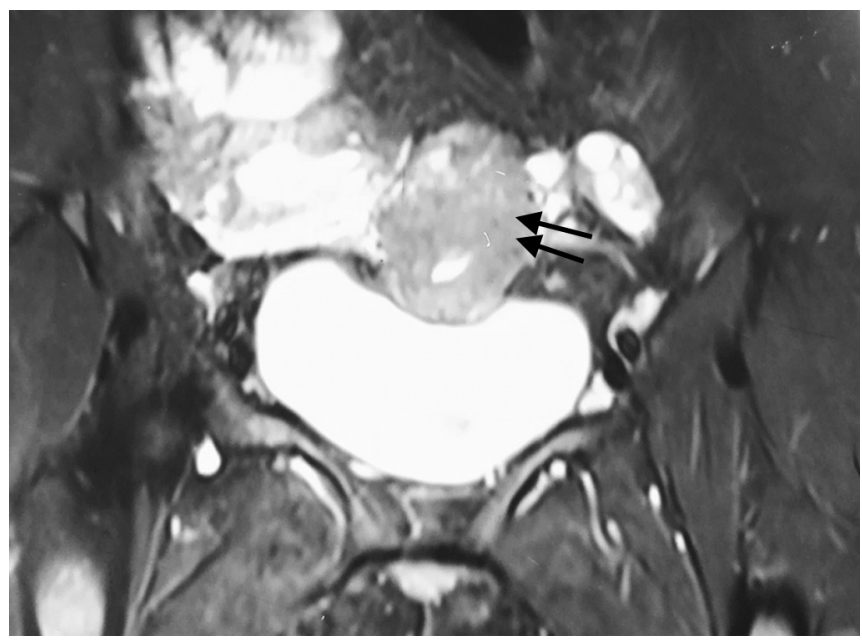

Fig. 1: MRI picture showing the endometriotic lesion indenting dome of bladder

() The Author(s). 2020 Open Access This article is distributed under the terms of the Creative Commons Attribution 4.0 International License (https://creativecommons. org/licenses/by-nc/4.0/), which permits unrestricted use, distribution, and non-commercial reproduction in any medium, provided you give appropriate credit to the original author(s) and the source, provide a link to the Creative Commons license, and indicate if changes were made. The Creative Commons Public Domain Dedication waiver (http://creativecommons.org/publicdomain/zero/1.0/) applies to the data made available in this article, unless otherwise stated. 


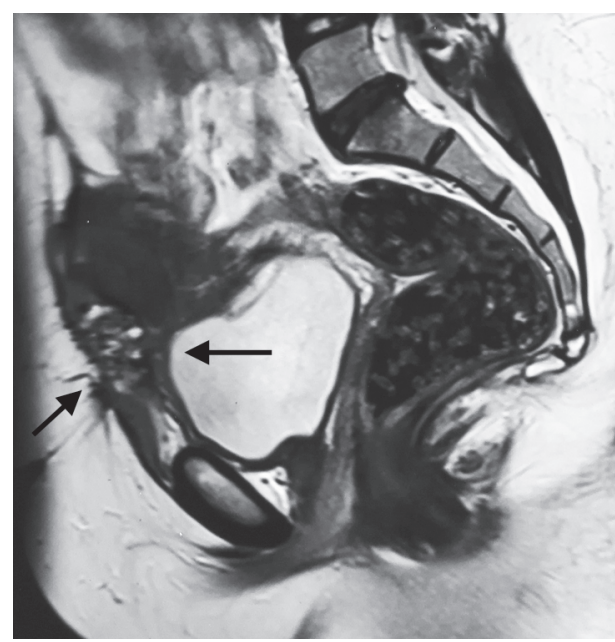

Fig. 2: MRI showing the endometriosis within the rectus abdominis muscle

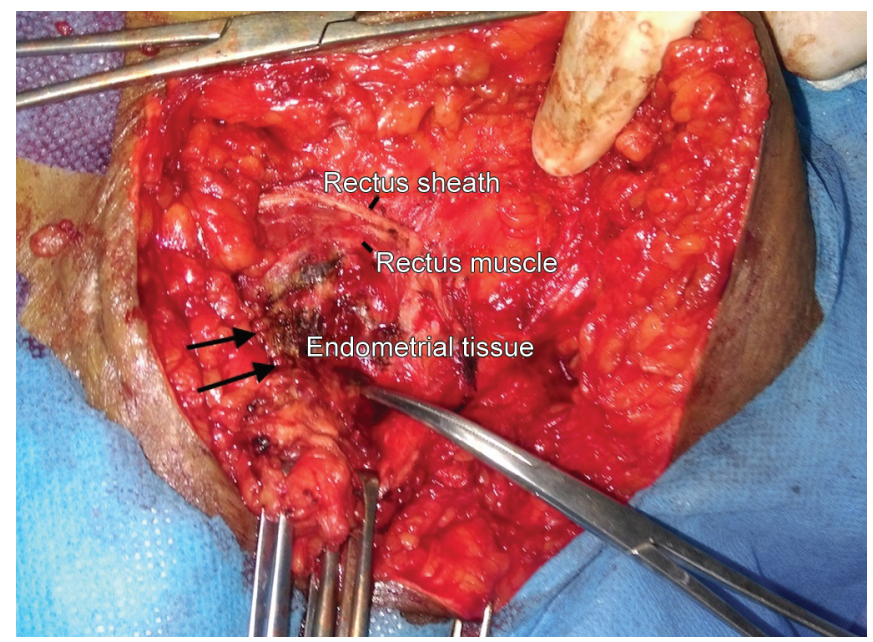

Fig. 4: Intraoperative picture showing the bluish endometriotic glands

regional anesthesia, the cesarean section scar was excised with the exploration of the underlying rectus muscle. Indurated tissue was seen beneath the scar, with interspersed bluish glandular endometriotic tissue which extended through the rectus muscle (Figs 3 and 4) and was adherent to the anterior wall of the uterus and compressing the dome of bladder. This tissue was excised widely including a rim of surrounding normal muscle to avoid the incomplete excision. The total abdominal hysterectomy was done (Fig. 5). The resultant defect in the rectus muscle and sheath was repaired with a $10 \times 7.5 \mathrm{~cm}$ polypropylene mesh. Histopathology of the excised specimen was reported as bundles of skeletal muscle tissue invaginated by endometrial glands with stromal cells (Figs 6 and 7). Patient recovered uneventfully postoperatively, is symptom-free for the past three months, and is on regular follow-up.

\section{Discussion}

Endometriosis as a disease can occur in pelvic and extrapelvic sites. While pelvic endometriosis is the generally observed site of occurrence, extrapelvic endometriosis is extremely rare, with bowel,

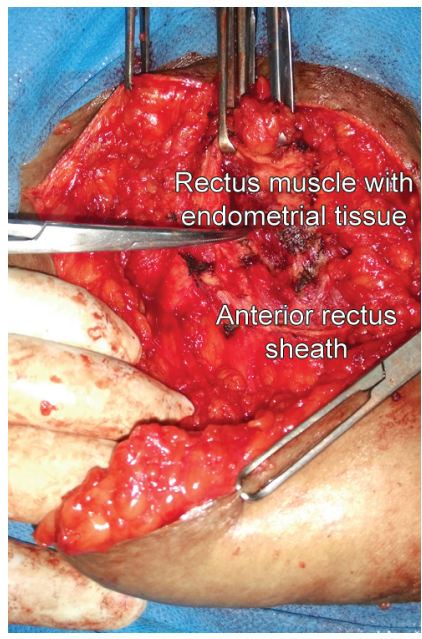

Fig. 3: Intraoperative picture showing the endometriotic tissue within the rectus abdominis

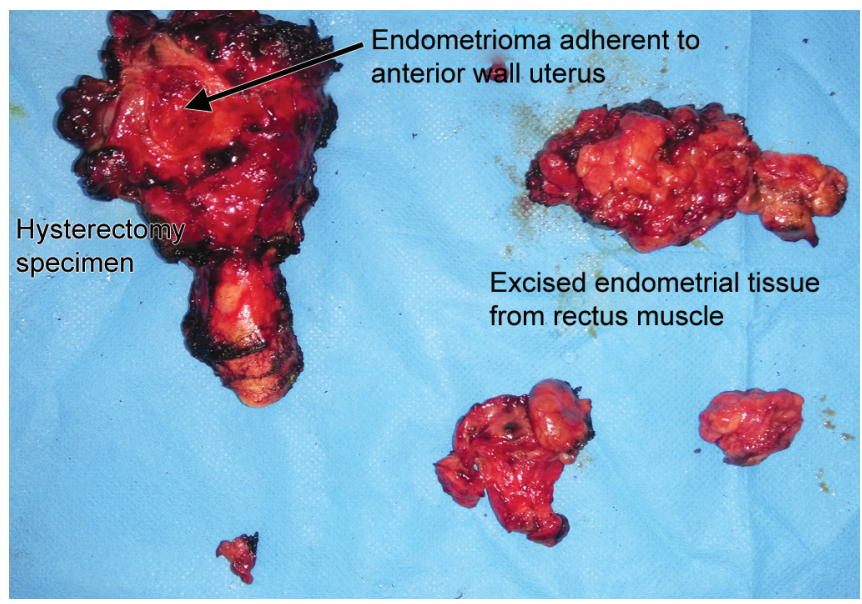

Fig. 5: Postoperative specimen showing the area of adherence of endometriotic tissue to the anterior surface of uterus

umbilicus, abdominal scars, pleura, and lung being some of the reported sites. ${ }^{1}$ Among these, endometriosis of the rectus muscle is extremely rare with the first case being reported by Amato and Levitt in $1984 .^{2}$ Since then, only about 20 cases have been reported in the literature. ${ }^{3}$ In the case series reported by Giannella et al. ${ }^{4}$ of 18 reported cases of the rectus muscle endometriosis, the average size was $4 \times 4 \mathrm{~cm}$ and it was prevalent in the premenopausal age group of 27-42 years. The largest size reported in this review was from Kocakusak et al., ${ }^{5}$ of $7 \times 7 \mathrm{~cm}$ and $7 \times 5 \mathrm{~cm}$ in a 37-year-old and a 27-year-old, respectively. Our patient had a comparable size to this, with the MRI imaging reporting a size of $6 \times 3.3 \mathrm{~cm}$. Hence, we presume this to be one of the large-sized rectus muscle endometriomas to be reported till date. FNA has not been reported to be of much significance; ${ }^{3}$ hence, we followed the CT and MRI imaging protocols for preoperative diagnosis in this patient.

Endometrioma has an estrogen-dependent growth accounting for the cyclical pain experienced by patients during menses. There are various theories attributed to the development of endometriotic tissue. Some of these are the metaplasia theory, retrograde menstruation, vascular or lymphatic metastasis, and the mechanical implantation theory. ${ }^{4}$ Though the theory of mechanical 


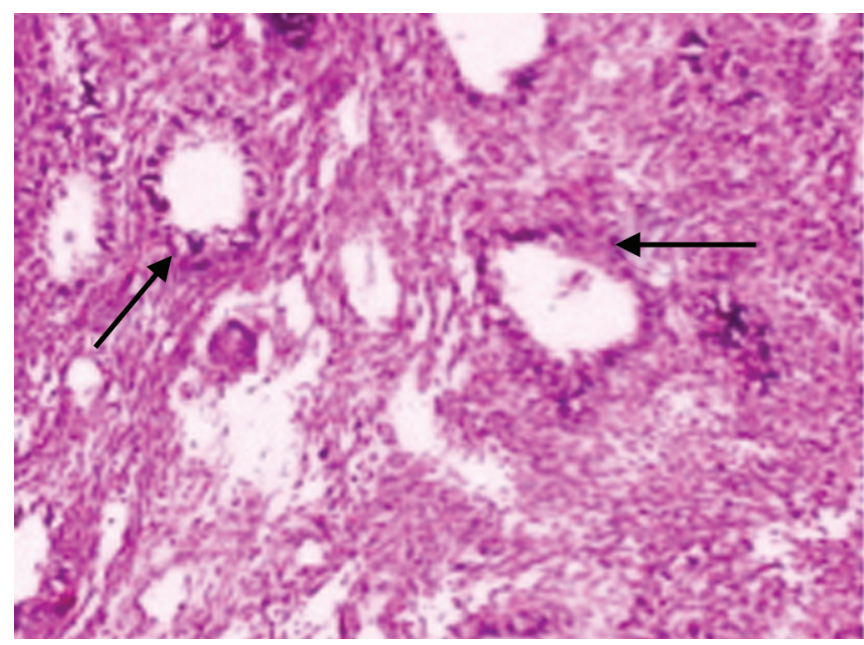

Fig. 6: Histopathological picture showing bundles of skeletal muscle fibers invaginated by endometrial glands and stroma-low-power view

implantation gives a logical explanation for the pathogenesis and its predominant occurrence in abdominal scars, it does not explain the implantation during a cesarean section since pregnancy is a progesterone-predominant phase which should negatively influence the implantation and growth of endometriotic tissue. ${ }^{4}$ Thus, the phenomenon of endometriosis can be satisfactorily explained only by a combination of mechanical and hormonal factors.

Most patients experience pain over the site of implantation as the primary symptom, usually at the site of previous surgery accounting for the scar tenderness. Among the 18 reported cases of rectus muscle endometriosis reviewed by Giannella et al., ${ }^{4}$ the predominant presenting symptoms of patients were pain with swelling (83\%), swelling (11\%), or pain alone (5\%). Most patients fail to notice the cyclical nature of pain and thus get treated in OPDs for adhesive colic or non-specific pain. Clinical diagnosis is usually inaccurate, and it is difficult to differentiate from other diagnoses, such as, abscess, hematoma, and stitch granuloma. Imaging is usually done by an ultrasonogram initially, which would reveal a heterogeneous textured lesion with hyperechoic internal echoes. ${ }^{5}$

Ultrasonogram rarely clinches the diagnosis; hence, a CT or MRI is usually done to look for solid heterogeneously enhancing lesions with irregular margins. In this case, a strong clinical suspicion with the imaging findings helped us to arrive at a preoperative diagnosis.

The role of serum markers in the preoperative diagnosis of endometriosis has been investigated by various authors. Luisi et al. ${ }^{6}$ reported the high levels of CA125 in endometriosis patients. Lermann et al. ${ }^{7}$ reported lower levels of high-sensitivity CRP in women with endometriosis compared to normal women. Another study by Lemos et al. ${ }^{8}$ found out that the levels of anti-Mullerian hormone were decreased in women with endometriosis. The latest serum marker under study is follistatin, which shows a remarkable increase in levels in patients with endometriosis with better sensitivity than CA125. ${ }^{9}$ However, none of these markers have been found to have influenced the diagnostic armamentarium of endometriosis in a convincing way and are yet to be fully validated. ${ }^{3,4}$

Besides having a poor acceptance rate from patients owing to its side effect profile, medical treatment for endometriosis is usually ineffective when given as a primary therapy. Danazol and

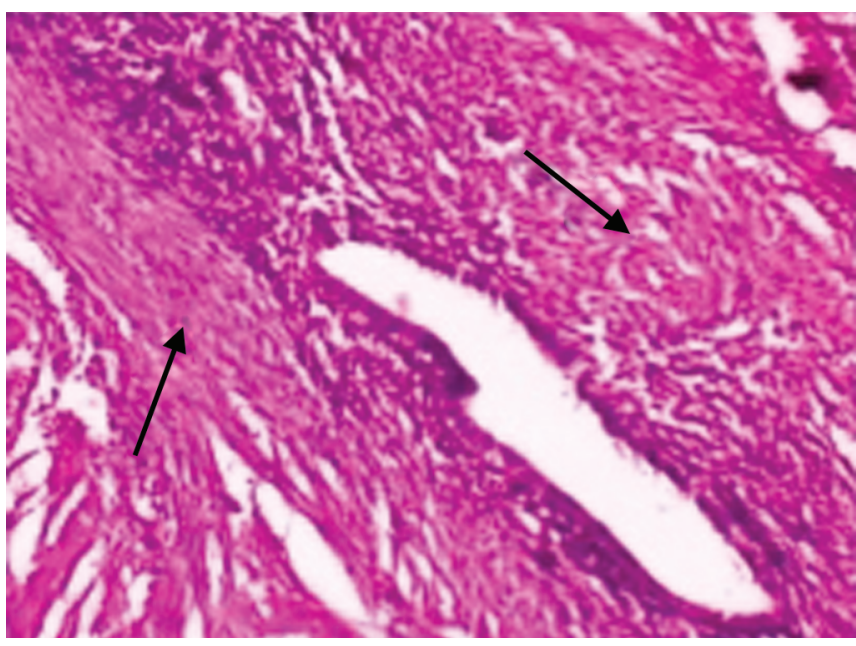

Fig. 7: Histopathological picture showing bundles of skeletal muscle fibers invaginated by endometrial glands and stroma-high-power view

progesterone are the drugs of choice. ${ }^{10}$ Thus, it is reserved for patients unfit for a surgical therapy, or it is used as an adjuvant therapy after the surgical excision of the endometriotic tissue to prevent recurrence. ${ }^{3}$ Surgical treatment of choice is the wide excision of the lesion so as not to leave out any residual tissue to avoid recurrence. Intraoperative spillage onto the normal territory must be avoided. ${ }^{4}$ The repair of the residual defect after the excision of the endometriotic tissue is important. This can be done either by darning repair with non-absorbable sutures or with a prosthetic mesh. So far, only four cases have been described in the literature where a prosthetic mesh has been used to repair the residual defect, 4 out of which two cases had a defect more than $5 \mathrm{~cm}$. In our case, there was a large defect in the rectus muscle with the loss of a considerable portion of the anterior rectus sheath. A polypropylene mesh of size $10 \times 7.5 \mathrm{~cm}$ was grafted as an onlay onto the defect.

\section{Conclusion}

Our case is one of the largest reported cases of the rectus muscle endometriosis repaired by a prosthetic mesh. This rare disease, although difficult to diagnose preoperatively, must be included in the differential diagnosis of abdominal pain in a menstruating woman. With strong clinical suspicion and proper preoperative imaging, a wide local excision of the lesion with adequate follow-up for recurrence would suffice as the satisfactory line of management.

\section{References}

1. Karaman H, Bulut F, Özaşlamacı A. Endometriosis externa within the rectus abdominis muscle. Turkish J Surg 2014;30(3):165-168. DOI: 10.5152/UCD.2014.2035.

2. Amato M, Levitt R. Abdominal wall endometrioma: CT findings. J Comput Assist Tomogr 1984;8(6):1213-1214. DOI: 10.1097/00004728198412000-00040.

3. Ozkan OF, Cikman O, Kiraz HA, et al. Endometrioma localized in the rectus abdominis muscle: a case report and review of literature. $A B C D$ Arquivos Brasileiros de Cirurgia Digestiva (São Paulo) 2014;27(3): 230-231. DOI: 10.1590/s0102-67202014000300019.

4. Giannella L, La Marca A, Ternelli G, et al. Rectus abdominis muscle endometriosis: case report and review of the literature. J Obstet Gynaecol Res 2010;36(4):902-906. DOI: 10.1111/j.14470756.2010.01236.x. 
5. Kocakusak A, Arpinar E, Arikan S, et al. Abdominal wall endometriosis: a diagnostic dilemma for surgeons. Med Princ Pract 2005;14(6):434437. DOI: $10.1159 / 000088118$

6. Luisi S, Gabbanini M, Sollazzi S, et al. Surgical scar endometriosis after cesarean section: a case report. Gynecol Endocrinol 2006;22(5):284285. DOI: 10.1080/09513590600777578.

7. Lermann J, Mueller A, Körber F, et al. Evaluation of high-sensitivity $\mathrm{C}$-reactive protein in comparison with $\mathrm{C}$-reactive protein as biochemical serum markers in women with endometriosis. Fertil Steril 2009;93(7):2125-2129. DOI: 10.1016/j.fertnstert.
8. Lemos NA, Arbo E, Scalco R, et al. Decreased anti-Müllerian hormone and altered ovarian follicular cohort in infertile patients with mild/ minimal endometriosis. Fertil Steril 2008;89(5):1064-1068. DOI: 10.1016/j.fertnstert.2007.04.048.

9. Florio $\mathrm{P}$, Reis FM, Torres PB, et al. High serum follistatin levels in women with ovarian endometriosis. Hum Reprod 2009;24(10):2600-2606 DOI: 10.1093/humrep/dep195.

10. Zararia AN, Goverdhan NA, Patil RT. Post caesarean scar endometriosis. JCR 2015;5:401-405. DOI: 10.17659/01.2015.0103. 